\title{
Efectos del Huracán Paloma sobre los pastos marinos del archipiélago Jardines de la Reina, Cuba
}

\author{
Mayrene Guimarais, Adán Zúñiga, Fabián Pina \& Felipe Matos \\ Centro de Investigaciones de Ecosistemas Costeros, Cayo Coco, Morón. CP 69400. Ciego de Ávila. Cuba; \\ mayrene@ciec.fica.inf.cu, adan@ciec.fica.inf.cu,fabian@ciec.fica.inf.cu,fmatos@ciec.fica.inf.cu
}

Recibido 15-VI-2012. Corregido 30-X-2012. Aceptado 28-XI-2012.

\begin{abstract}
Hurricane Paloma's effects on seagrasses along Jardines de la Reina archipelago, Cuba. Seagrasses are one of the most important coastal ecosystems since they promote organic matter flow, nutrient cycling, food availability and refuge. Until now, reports on damages caused by storms and hurricanes on seagrass beds are uncommon and highly variable. The seagrass meadows of the East end of Jardines de la Reina archipelago were surveyed from Nov. $2^{\text {th }}$ to Dec. $5^{\text {th }}$ of 2008 , in order to determinate the effects from the passing of Hurricane Paloma: a category three storm on the Saffir-Simpsom scale. A rapid field assessment of the affected areas was carried out using the manta tow technique. In six sites, seagrass was quantitatively evaluated using a $15 \mathrm{~cm}$ diameter core (four sampling units per site) and shoot density was calculated. Remote sensing techniques were used to estimate seagrass cover. To estimate the percentage of affected areas, a Region of Interest (ROI) was first created over a Landsat image. The percentage of seagrass affected within the ROI was estimated through direct georeferentiation of the contours of the damaged area and with a comparison to the total seagrass extension. To find possible explanations for damages, a false colour image was created using the near infrared band, to highlight the differences between emerged and submerged zones. The direction of winds was estimated using ArcGis 9.2 creating circular buffers, from the centre of the hurricane and generating lines tangent to the buffers. Seagrass in the region was dominated by the angiosperm Thalassia testudinum. Regional mean density was $1321 \pm 721$ shoots $/ \mathrm{m}^{2}$, a value regarded as high for the Caribbean area. Seagrass meadows were partly affected by sediment accumulation on the shoots of $T$. testudinum and uprooting rhizomes. The $7.6 \mathrm{~km}^{2}$ disturbed area represented $1 \%$ of the total seagrass area. Other sites, closer to the centre of the hurricane, did not show any damages on the marine vegetation. The keys location with respect to the hurricane track was the most likely cause of the effects. To the North of the affected area there is an opening among the keys where the generation of waves, currents and turbulence could have occurred. Three years after the hurricane event, both vegetation cover loss and silt re-colonization by macroalgae species were observed within the affected area, by showing a patchily-vegetated landscape. This site is currently undergoing a temporal succession whose assessment demands a monitoring scheme, that will provide interesting information to document its future evolution and responsiveness against upcoming natural or anthropogenic events. Rev. Biol. Trop. 61 (3): 1425-1432. Epub 2013 September 01.
\end{abstract}

Key words: seagrasses, hurricane Paloma, disturbance, Thalassia testudinum, Jardines de la Reina Archipelago.

Los pastos marinos son los principales y más valiosos ecosistemas costeros de la mayoría de los fondos blandos del mundo (McRoy \& Helferrich 1977, 1980). La pérdida de pastos marinos altera el flujo de materia orgánica, ciclo de nutrientes y cadena alimenticia a lo largo del pastizal y hacia los ecosistemas costeros adyacentes (Bach et al. 1986, Preen et al. 1995). La pérdida de estos importantes procesos ecológicos eventualmente conduce a desastres incluyendo colapso de pesquerías, degradación de la calidad del agua y declinación de otros recursos vivos (De Jonge \& De Jonge 1992).

Desde los inicios de la década del 70, están sucediendo declinaciones en la distribución y abundancia de los pastos marinos (Cambridge et al. 1986, Dennison et al. 1993, Short \& 
Wyllie-Echeverria 1996). Las descripciones de disturbios naturales, provocados por tormentas o huracanes, sobre las comunidades de pastos marinos son altamente variables. Numerosos estudios informan pérdidas de pastos marinos como resultado de desenterramiento por fuerzas físicas o enterramiento por deposición de sedimentos (Kenyon \& Poiner 1987, Fonseca et al. 2000, Fourqurean \& Rutten 2004, CruzPalacios \& Van Tussenbroek 2005, Ridler et al. 2006, Steward et al. 2006, Bell et al. 2008, Van Tussenbroek et al. 2008).

El tiempo requerido para que un pasto marino se recupere de un disturbio está en función de su magnitud. Sin embargo, los efectos de grandes perturbaciones causadas por ciclones tropicales podrían persistir por muchas décadas. Los estudios sobre la dinámica de recuperación puede tornarse muy difíciles por la larga escala de tiempo, pero el entendimiento de la tasa y los mecanismos de recuperación de los pastizales es importante no sólo con fines científicos, sino para el manejo del ecosistema ante daños o con fines de restauración (Fourqurean \& Rutten 2004).

Según criterios de Appeldoorn \& Lindeman (2003), el archipiélago de Jardines de la Reina (AJR) es considerado la mayor reserva marina del Caribe, y una de las áreas más saludables y diversas de dicha región (Linton et al. 2002). En el archipiélago existen extensas áreas de pastos marinos, pero pocas investigaciones desarrolladas sobre estos. De igual manera ocurre con la información sobre las afectaciones que sufre este ecosistema por el paso de huracanes por la plataforma insular cubana.

El Atlántico, Golfo de México y el Caribe constituyen un área oceánica activa en cuanto a huracanes tropicales, puesta de manifiesto a través de sólidas informaciones cronológicas en el área (Landsea 1993, Ortíz 1994). Adicionalmente, resulta importante señalar que Cuba, en su condición de archipiélago, recibe grandes afectaciones por este tipo de fenómenos (Limia et al. 2003).

El huracán Paloma constituyó un fenómeno de gran interés para Cuba debido a la incidencia negativa a su paso (Ballester \&
Rubiera, 2009), aunque el mayor número de información referida a los impactos aparecen mayormente centrados en sectores socioeconómicos. Este sistema, catalogado con categoría tres en la escala Saffir-Simpsom, azotó el AJR con vientos huracanados desde las 4:00 PM (21:00 UTC) hasta las 7:00 PM (00:00 UTC) del 8 de noviembre del 2008. El paso de este evento meteorológico, brindó la posibilidad de cuantificar la abundancia y extensión de los pastos marinos y de evaluar las alteraciones que su trayectoria dejó sobre los mismos, a la vez que permitió dar seguimiento a la evolución futura del ecosistema en una zona con elevadas condiciones de protección ambiental.

\section{MATERIALES Y MÉTODOS}

El área de estudio se localiza en el extremo oriental del Parque Nacional Jardines de la Reina (PNJR), al SE de Cuba (20³0' N $78^{\circ} 55^{\prime} \mathrm{W}$ y $20^{\circ} 50^{\prime} \mathrm{N}-78^{\circ} 20^{\prime} \mathrm{W}$ ), sitio donde ocurrió el primer punto de contacto del Huracán Paloma con el territorio cubano (Fig. 1). El viento en la zona de interés osciló entre

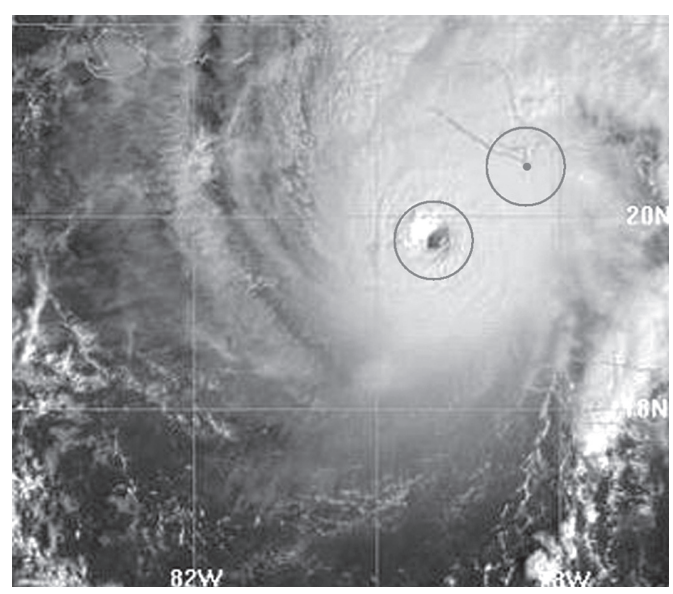

Fig. 1. Huracán Paloma. Imagen VIS del satélite GOES-12 del 8 noviembre 2008, 13: 45 UTC. Fuente: www.insmet. cu/. Nota: El punto representa la posición más cercana al área de estudio y el círculo rojo el anillo de viento máximo. Fig. 1. Paloma Hurricane. VIS Image satellite GOES, November 8th to 12th 2008, 13: 45 UTC. Source: www. insmet.cu/. Note: The point represent the closest position to the study area and the red circle, the zone with maximum speed of winds. 
178 y $195 \mathrm{~km} / \mathrm{h}$. La intensidad de $195 \mathrm{~km} / \mathrm{h}$ (categoría tres) fue estimada por el valor del viento máximo (118 nudos) a nivel de vuelo observado por un avión de reconocimiento a las 4:28 PM (21:28 UTC), justo al sudeste de Cayo Cabeza del Este.

Del 29 de noviembre al 5 de diciembre 2008 se evaluaron los efectos de este huracán sobre los pastos marinos. Se llevó a cabo una evaluación rápida de campo mediante recorridos con arrastre (manta tow) (Roger et al. 1994) lo que permitió determinar las áreas bajo disturbios desde Los Hierros hasta Boca Piedra Chiquita. En seis sitios: Las Varas, Juan Grin, La Bola, Víveres, Cachiboca y Boca Piedra Chiquita se evaluó cuantitativamente el pasto marino mediante nucleador de $15 \mathrm{~cm}$ de diámetro (cuatro unidades de muestreo por sitio) (CARICOMP 1994, 2001). Las muestras fueron lavadas con agua de mar para eliminar el sedimento, envasadas en bolsas de plástico y etiquetadas. En el laboratorio, se contó el número de vástagos por unidad de muestreo y se calculó la densidad. Todas las estaciones se referenciaron por medio de un GPS72 (Garmin), con precisión entre tres y cinco metros.

Para estimar el porcentaje de las áreas de pastos marinos afectadas y el área ocupada por cada biotopo, se creó una Región de Interés (ROI), sobre la imagen Landsat7 (Enhanced Thematic Mapper plus (ETM+) utilizando el software ENVI 3.6. La ROI se definió teniendo en cuenta la zona hasta donde llegaron los vientos huracanados y parte de tormenta tropical. El recorte realizado fue procesado en ArcGis 9.2, donde inicialmente se efectuó una clasificación no supervisada con nueve clases espectrales, que fueron posteriormente verificadas in situ con la comprobación de más de cinco puntos por cada clase, lo que permitió generar el mapa de los biotopos marinos y estimar la extensión de los pastos. El área ocupada por cada biotopo fue calculada, a partir, de la sumatoria de los pixeles con rangos espectrales similares por clase. El porcentaje de afectación de los pastos en la ROI fue estimado por la georeferenciación directa de los contornos del área dañada y su comparación posterior con la extensión total del pasto.

Para encontrar posibles explicaciones a los disturbios, se creó una imagen de falso color utilizando la banda del infrarrojo cercano para resaltar las diferencias entre las zonas emergidas y sumergidas, y se realizaron comprobaciones de campo. Sobre la imagen compuesta, se delimitó la zona de vientos con categoría de huracán. La dirección de los vientos fue estimada con ArcGis 9.2 mediante la elaboración de buffers circulares, a partir del centro del huracán en el momento del paso por el área, generando líneas tangenciales a los buffers. Los datos de la marea se obtuvieron utilizando el Software WXTide32 versión 2.4 para la estación mareográfica más cercana a la zona (Manzanillo, Golfo de Guacanayabo).

En Octubre 2011 se visitó nuevamente el área afectada y se realizó una evaluación cualitativa, mediante recorrido libre. Los cambios en el paisaje marino se documentaron con fotografías.

\section{RESULTADOS}

En la zona estudiada, los pastos marinos estuvieron dominados por la angiosperma Thalassia testudinum Banks ex König, con densidades de $1220 \pm 446$ vástagos $/ \mathrm{m}^{2}$ en Juan Grin y hasta $2235 \pm 410$ vástagos $/ \mathrm{m}^{2}$ en Boca Piedra Chiquita, para una media regional de $1321 \pm 721$ vástagos $/ \mathrm{m}^{2}$. Únicamente apareció Syringodium filiforme Kützing in Hohenacker en Boca Piedra Chiquita (Fig. 2).

La extensión de los pastos marinos dentro de la ROI alcanzó los $750 \mathrm{~km}^{2}$, considerando la sumatoria de las áreas que ocupan el pasto denso y poco denso. La distribución de los rangos de color de los píxeles clasificados como biotopos con presencia de fanerógamas, muestra la dominancia de los pastos marinos sobre el resto de los tipos de fondo. Los pastos (denso y poco denso) conformaron el 54\% de todos los biotopos identificados en el área, sin tomar en consideración los píxeles no clasificados (139255) y los correspondientes a la zona profunda (0-16) (Fig. 3 y 4). 


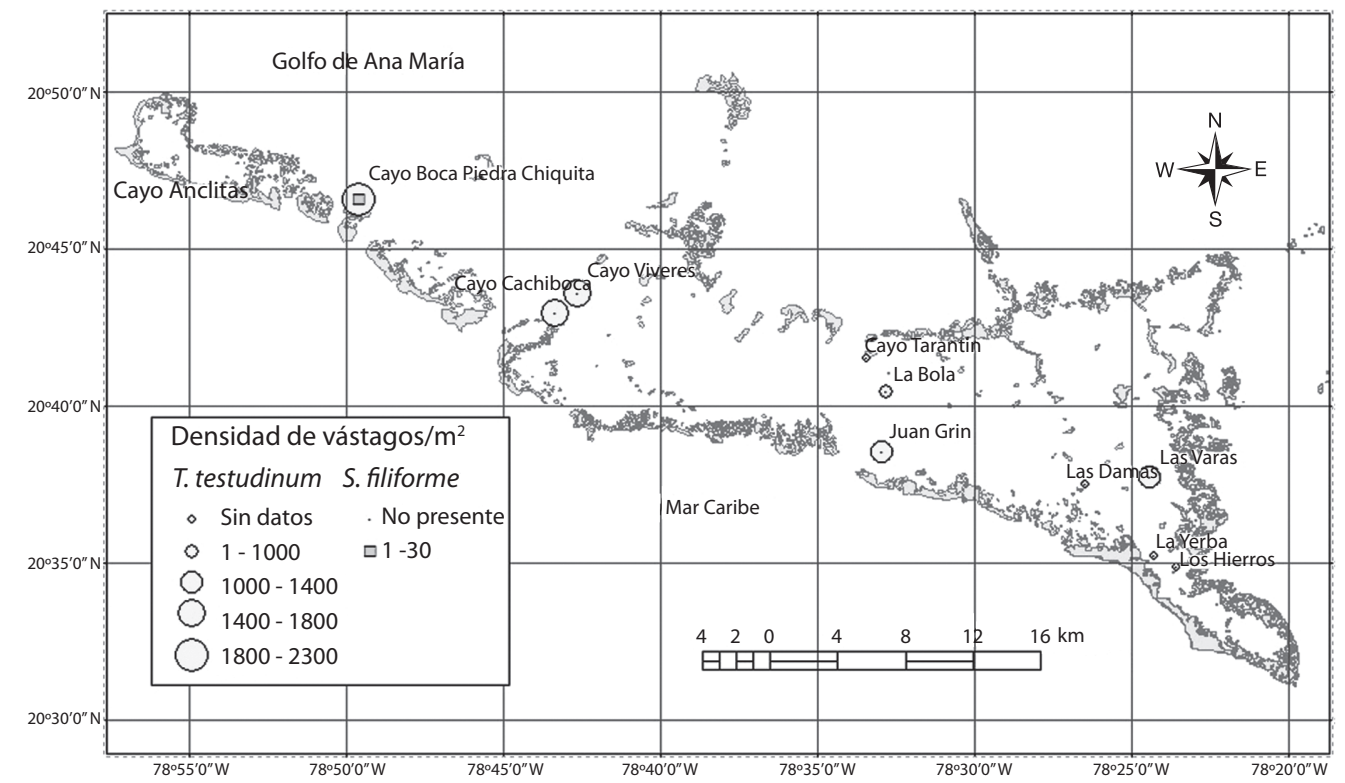

Fig. 2. Densidad de vástagos de Thalassia testudinum y Syringodium filiforme.

Fig. 2. Shoots density of Thalassia testudinum and Syringodium filiforme.

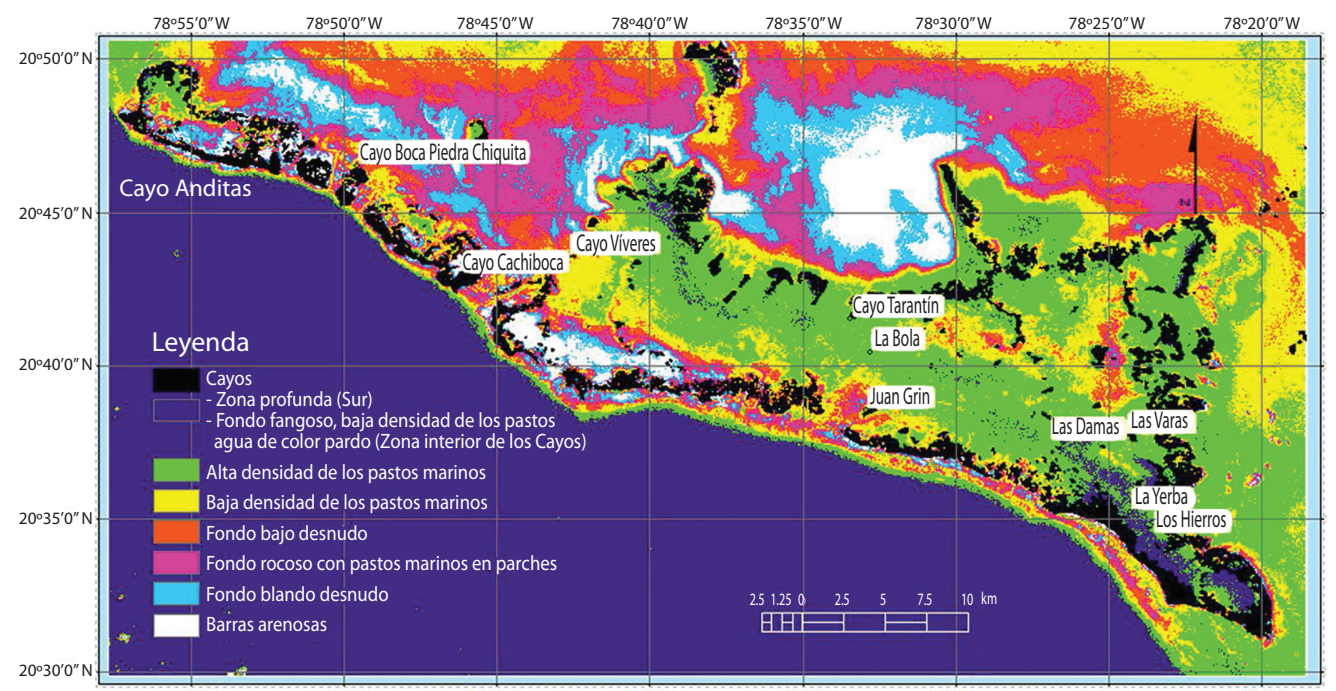

Fig. 3. Biotopos identificados en el área de estudio.

Fig. 3. Biotopes identified in the study area.

El área bajo influencia directa de los vientos huracanados se extendió desde el extremo oriental del archipiélago hasta $4 \mathrm{~km}$ al este de Víveres. Se observó afectación del pasto marino en el tramo comprendido entre La Bola y
Tarantín, localizados a 22 y $24 \mathrm{~km}$ respectivamente, de la posición más cercana del centro del huracán. El disturbio se observó como la acumulación de sedimentos sobre los vástagos de T. testudinum o por el desenterramiento de 


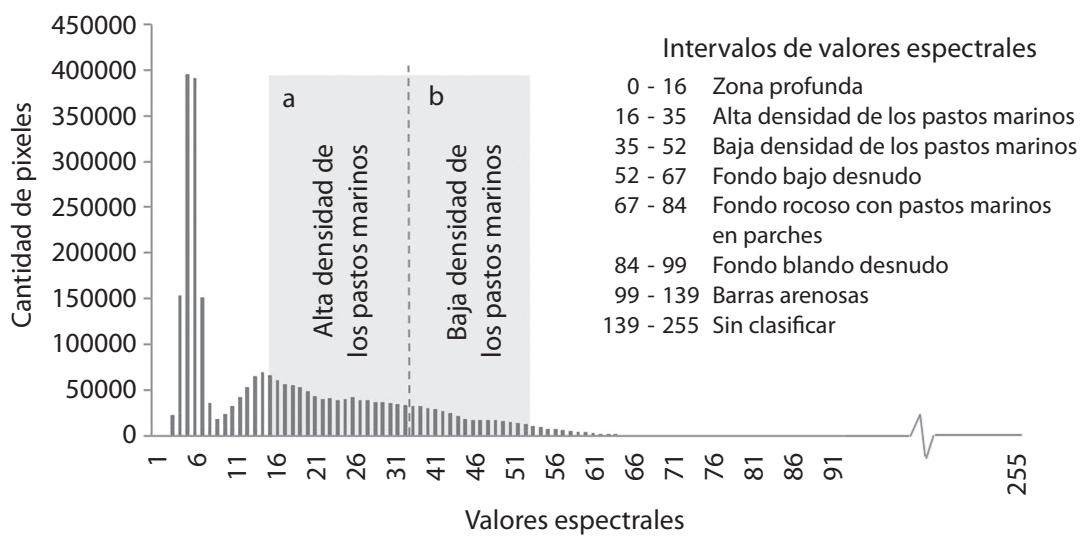

Fig. 4. Dominancia de los fondos cubiertos por pastos marinos en la ROI. Nota: a-Rango de pixeles pertenecientes al biotopo Alta densidad de pastos marinos (área $425 \mathrm{~km}^{2}$ ); b- Rango de pixeles pertenecientes al biotopo Baja densidad de pastos marinos (área $325 \mathrm{~km}^{2}$ ).

Fig. 4. Dominance of bottoms covered by seagrass inside the ROI. Note: a- Range of pixels correspond to High-density seagrass meadows (area: $425 \mathrm{~km}^{2}$ ); b- Range of pixels correspond to Low-density seagrass meadows (area: $325 \mathrm{~km}^{2}$ ).

rizomas. Se calculó un área afectada de $7.6 \mathrm{~km}^{2}$, que representó el $1 \%$ del total de pastos marinos estimados para la ROI (Fig. 5).

Los valores de marea en el momento del paso del huracán fueron máximos $(0.65 \mathrm{~m})$. Sitios más cercanos al centro del meteoro, como Los Hierros $(2.7 \mathrm{~km})$ y la Yerba $(4.1 \mathrm{~km})$ no mostraron afectaciones en la vegetación marina. Por otra parte, los pastos marinos ubicados en el área bajo influencia de vientos de tormenta tropical no presentaron afectaciones.

Prácticamente tres años después del paso del huracán, se visitó nuevamente el área afectada, en la que se encontró una extensa área, aproximadamente un $60 \%$, cubierta por algas, fundamentalmente rizofíticas de los géneros Caulerpa, Halimeda y Penicillus y flotantes del género Laurencia, principalmente (Fig. 6 a y b). La evolución del sitio apunta hacia una pérdida de vegetación y re-colonización del sedimento por especies de macroalgas, mostrándose el paisaje con vegetación por parches.

\section{DISCUSIÓN}

Las densidades de $T$. testudinum fueron superiores a las encontradas en la costa norte de Ciego de Ávila por Clero-Alonso et al.
(2006) y se consideran altas para la zona del Caribe al comparar con datos de CARICOMP (2004). Estas densidades, denotan condiciones ambientales idóneas para la extensión del ecosistema, quizás determinadas por el aislamiento del archipiélago y por ende el alto grado de conservación del mismo. La presencia de $T$. testudinum como especie clímax corrobora la estabilidad del área.

Una posible explicación de que los pastos más cercanos al centro del huracán no hayan sufrido afectaciones y que otros más alejados sí, puede ser la protección que ofrecieron los cayos. En el extremo oriental del área, los cayos se encuentran en una disposición que puede haber influido en la atenuación del oleaje y de la velocidad de las corrientes.

Al norte de la zona afectada, la distribución de los cayos posee una abertura que puede haber facilitado y reforzado la generación de oleaje y corriente, provocados por la acción de los vientos que soplaron en este sitio del NNE, durante la posición más cercana del centro del huracán. Esta explicación puede verse reforzada por las acumulaciones de sedimento fango arenoso encontradas sobre el área afectada, que deben haber sido arrastrados desde la zona norte de los cayos, donde el fondo es desnudo. 


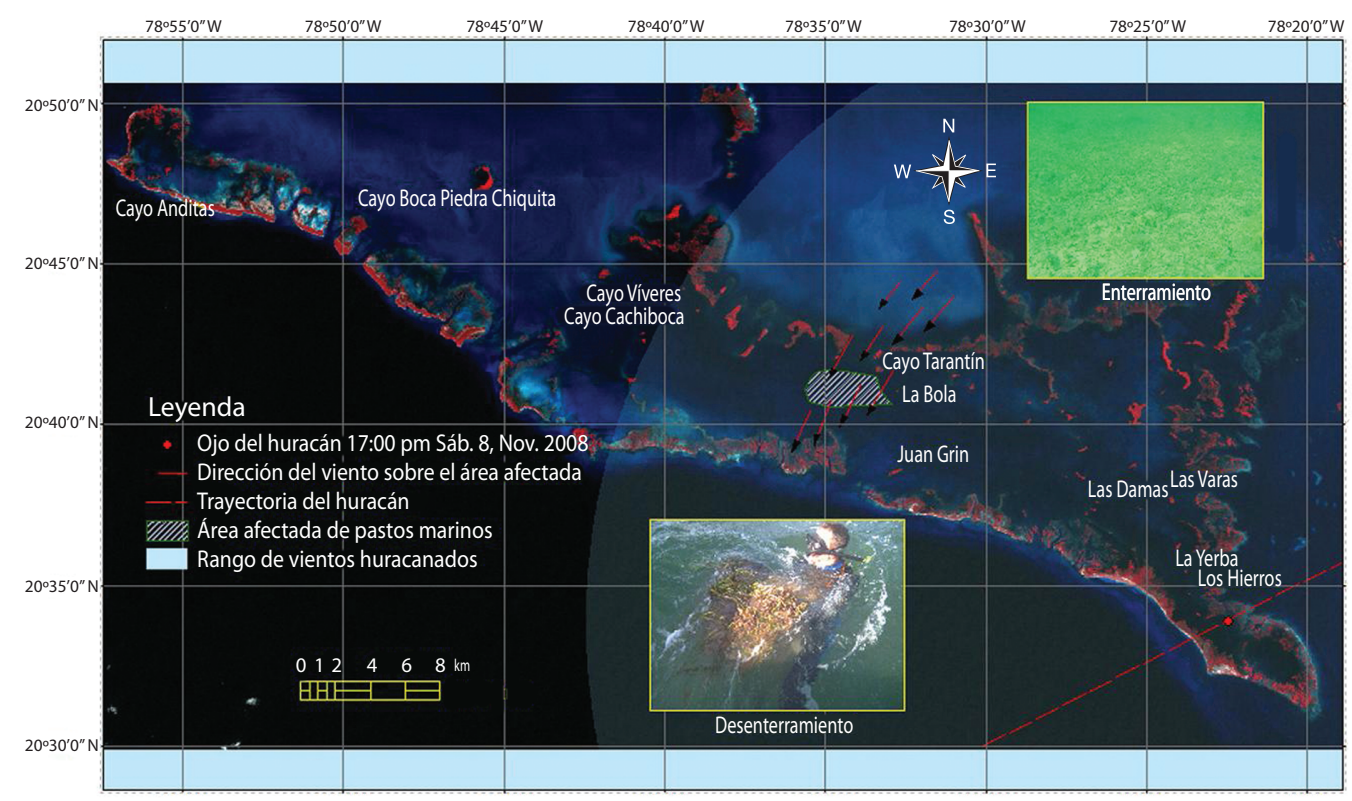

Fig. 5. Área de pastos marinos afectados por enterramiento y erosión. Incidencia y dirección de los vientos sobre el área bajo disturbio.

Fig. 5. Area of seagrass affected by sediment deposition and uprooting. Incidence of winds on the disturbed area.
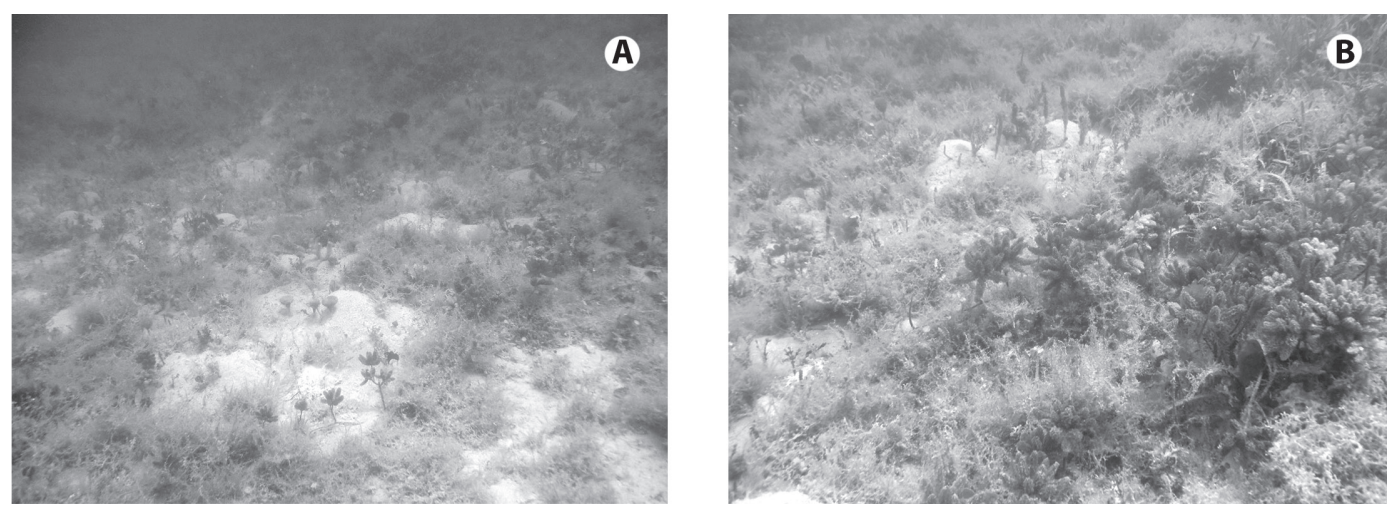

Fig. 6a, b. Colonización del área afectada por algas rizofíticas y flotantes.

Fig. 6a, b. Affected area by colonization of rhizophytic and drift algae.

La ocurrencia de procesos locales con vientos de mayor intensidad dentro de la propia zona de vientos huracanados, no se descarta como una posibilidad de haber provocado el disturbio. Sin embargo, parece más probable la explicación relacionada con la disposición de los cayos, si se toma en consideración además, que los valores de la marea fueron máximos, sumado como otro factor la sobre elevación del nivel del mar que se genera en un huracán de esta envergadura.

Los eventos que generan aumento de la corriente, oleaje y consecuentemente fuerte turbulencia, provocan enterramiento y erosión de los pastos, lo que pudiera causar mortalidad de la vegetación en grado dependiente de la frecuencia de los mismos. El cambio observado después de tres años del disturbio, coincide con 
lo encontrado en el Sur de la Florida tras el paso del huracán Georges (Fourqurean \& Rutten 2004). Varios autores señalan diferentes destinos en la evolución o modificación del paisaje de este ecosistema después del paso de huracanes (Cruz-Palacios \& Van Tussenbroek 2005, Orth et al. 2006, Hall et al. 2006, Hammestrom et al. 2006, Bell et al. 2008). Lo encontrado concuerda con pérdida de la vegetación existente e incremento de los parches sin vegetación (Davis et al. 2004) y colonización temprana de algas (Fourqurean \& Rutten 2004).

Otros autores señalan que, las algas rizofíticas facilitan la recolonización de angiospermas marinas en sustratos desnudos (Williams 1990) y que también contribuyen con la estabilización del sustrato (Hammestrom et al. 2007). Tomando en consideración lo anterior, a tres años después del disturbio, el área se encuentra todavía en una sucesión temporal, por lo que no se descarta su posterior recuperación. Se hace oportuno evaluar el área afectada mediante un programa de monitoreo para documentar su evolución futura y ante nuevos episodios naturales y/o antrópicos.

\section{AGRADECIMIENTOS}

Los autores queremos agradecer a Gerardo Vera y Eduardo del Sol por su asistencia en el campo, a Eric Ceruto por su ayuda con la embarcación. A Giuseppe Omegna (Pepe), por apoyar las investigaciones a favor del PNJR. A Alain Parada por la revisión del inglés. Un agradecimiento especial a los revisores anónimos por sus valiosas sugerencias.

\section{RESUMEN}

Las descripciones de disturbios naturales, provocados por tormentas o huracanes, sobre las comunidades de pastos marinos son altamente variables. Del 29 de noviembre al 5 de diciembre 2008 se determinaron los efectos del huracán Paloma, categoría tres en la escala SaffirSimpsom, sobre los pastos marinos del extremo oriental del Archipiélago Jardines de la Reina. Se llevó a cabo una evaluación rápida de campo mediante recorridos con arrastre (manta tow) y se muestreo cuantitativamente en seis sitios. Se utilizaron técnicas de teledetección para estimar la extensión de los pastos. La densidad media regional fue de $1321 \pm 721$ vástagos $/ \mathrm{m}^{2}$, valor considerado como alto para la zona del Caribe. El pasto marino sufrió afectaciones parciales provocadas por acumulación de sedimentos sobre los vástagos de Thalassia testudinum o desenterramiento de rizomas. El área bajo disturbios fue de $7.6 \mathrm{~km}^{2}$ que representa el $1 \%$ del total de pastos marinos. Sitios más cercanos al ojo del huracán no mostraron afectaciones en la vegetación marina. La distribución de los cayos con respecto a la trayectoria del huracán se define como la causa más probable del patrón de afectación observado. Después de tres años del paso del huracán, la evolución del área afectada apunta hacia una pérdida de vegetación y re-colonización del sedimento por especies de macroalgas, se observa un paisaje con vegetación por parches. El sitio se encuentra en una sucesión temporal, por lo que se hace oportuno evaluar el área afectada mediante un programa de monitoreo para documentar su evolución futura y ante nuevos episodios naturales y/o antrópicos.

Palabras clave: pastos marinos, huracán Paloma, disturbios, Thalassia testudinum, archipiélago Jardines de la Reina.

\section{REFERENCIAS}

Appeldoorn, R.S. \& K.C. Lindeman. 2003. A CaribbeanWide Survey of Marine Reserves: Spatial coverage and Attributes of Effectiveness. Gulf Caribb. Res. 14: 139-154.

Bach, S., G. Thayer \& M. LaCroix. 1986. Export of detritus from eelgrass (Zostera marina) beds near Beaufort, North Carolina, USA. Mar Ecol. Prog. Ser. 28: 265-278.

Ballester, M. \& J. Rubiera. 2009. Temporada ciclónica de 2009 en el Atlántico Norte. Boletín Instituto de Meteorología.

Bell, S.S., M.S. Fonseca \& W.J. Kenworthy. 2008. Dynamics of a subtropical seagrass landscape: links between disturbance and mobile seed banks. Landscape Ecol. 23: 67-74.

Cambridge, M.A., A.W. Chiffings, C. Brittan, L. Moore \& A.J. Mccomb. 1986. The loss of seagrass in Cockburn Sound, Western Australia. II. Possible causes of seagrass decline. Aquat. Bot. 24: 137-148.

CARICOMP. 1994. CARICOMP Methods Manual-Level I. Manual of Methods for Mapping and Monitoring of Physical and Biological Parameters in the Coastal Zone of the Caribbean. University of the West Indies, Mona, Kingston, Jamaica: CARICOMP Data Management Center.

CARICOMP. 2001. CARICOMP Methods Manual. Levels I \& II. Methods for Mapping and Monitoring of Physical and Biological Parameters in the Coastal Zone of the Caribbean. Kingston, Jamaica y Florida, USA: CARICOMP Data Management Center. 
CARICOMP. 2004. The Caribbean Coastal Marine Productivity Program (CARICOMP), Annual Report. Caribbean Coastal Data Centre. Centre for Marine Sciences. University of the West Indies, Mona.

Clero Alonso, L., F. Pina-Amargós, L. Hernández-Fernández, F. Martín, D. Zúñiga, Sh. Cowling, A.K. Brady \& S. Caldwell. 2006. Biota acuática del norte de la provincia Ciego de Ávila, p. 182-194. In F. PinaAmargós (ed.). Ecosistemas costeros: biodiversidad y gestión de recursos naturales. Compilación por el XV Aniversario del CIEC. Sección I. Ecosistemas del norte de la provincia Ciego de Ávila. CIEC. CUJAE, La Habana, Cuba.

Cruz-Palacios, V. \& B.I. Van Tussenbroek. 2005. Simulation of hurricane-like disturbances on a Caribbean seagrass bed. J. Exp. Mar. Biol. Ecol. 324: 44-60.

Davis, S.E., J.E. Cablet \& D.L. Childers. 2004. Importance of storm events in controlling ecosystem structure and function in a Florida Gulf Coast Estuary. J. Coast. Res. 20: $1198-1208$

De Jonge, V.N. \& D.J. De Jonge. 1992. Role of tide, light, and fisheries in the decline of Zostera marina $\mathrm{L}$ in the DutchWadden Sea. Neth. Sea Res. Publ. Ser. 20: 161-176.

Dennison, W.C., R.J. Orth, K.A. Moore, J.C. Stevenson, V. Carter, S. Kollar, P.W. Bergstrom \& R.A. Batiuk. 1993. Assessing water quality with submerged aquatic vegetation. BioScience 161: 311-312.

Fonseca, M.S., W.J. Kenworthy \& P.E. Whitfield. 2000. Temporal dynamics of seagrass landscapes: a preliminary comparison of chronic and extreme disturbance events, p. 373-376. In G. Pergent, C. Pergent-Martini, M.C. Buia \& M.C. Gambi (eds.). Proceedings of the $4^{\text {th }}$ Internat Seagrass Biol Workshop. Corsica, France.

Fourqurean, J.W. \& L.M. Rutten. 2004. The impact of Hurricane Georges on soft-bottom, back reef communities: site-and species-specific effects in South Florida seagrass beds. Bull. Mar. Sci. 75: 239-257.

Hall, L.M., M.D. Hanisak \& R.W. Virnstein. 2006. Fragments of the seagrasses, Halodule wrightii and Halophila johnsonii, as potential recruits in Indian River Lagoon, Florida. Mar. Ecol. Prog. Ser. 310: 109-117.

Hammestrom, K.K., W.J. Kenworthy \& M.S. Fonseca. 2006. Seed bank, biomass, and productivity of Halophila decipiens, a deep water seagrass on the west Florida continental shelf. Aquat. Bot. 84: 110-120.

Hammerstrom, K., W. Kenworthy, P. Whitfield \& M. Merello. 2007. Response and recovery dynamics of seagrasses Thalassia testudinum and Syringodium filiforme and macroalgae in experimental motor vessel disturbances. Mar. Ecol. Prog. Ser. 345: 83-92.

Kenyon, R. \& I. Poiner. 1987. Seagrass and cyclones in the western Gulf of Carpentaria. CSIRO Fact Sheet No. 1, Cleveland, Queenland, Australia.

Landsea, C.W. 1993. Una Climatología de Huracanes Intensos (de Gran Magnitud) del Atlántico. Mon. Wea. Rev. 121: 1703-1713.
Limia, M., R. Vega \& R. Pérez. 2003. Climatología de los ciclones tropicales que han afectado a Cuba y sus provincias. En Memorias del X Congreso Latinoamericano e Ibérico de Meteorología "La meteorología y el desarrollo sostenible". La Habana, Cuba.

Linton, D., R. Smith, P. Alcolado, C. Hanson, P. Edwards, R. Estrada, T. Fisher, R.G. Fernández, F. Geraldes, C. Mccoy, D. Vaughan, V. Voegeli, G. Warner \& J. Wiener. 2002. Status of Coral Reefs in the Northern Caribbean and Atlantic Node of the GCRMN, p. $277-$ 302. In C. Wilkinson (ed.). Status of Coral reefs of the World. GCRMN Report. Institute of Marine Science, Townsville, Australia.

McRoy, C.P. \& C. Helferrich. 1977. Seagrass ecosystems: a scientific perspective. Marcel Dekker, New York, USA.

McRoy, C.P. \& C. Helferrich. 1980. Applied aspects of seagrasses, p. 297-343 In R.C. Phillips and C.P. McRoy (eds.). Handbook of seagrass biology: an ecosystem perspective. Garland STPM, New York, USA.

Orth, R.J., M.C. Harwell \& G.J. Inglis. 2006. Ecology of seagrass seeds and seagrass dispersal strategies, p. 111133. In T. Larkum, R. Orth \& C. Duarte (eds.). Seagrasses: Biology, Ecology and Conservation. Springer, Dordrecht, Netherlands.

Ortiz, R. 1994. Trayectorias de los ciclones tropicales del Atlántico. Instituto Cubano de Meteorología, CITMA, La Habana.

Preen, A.R., W.J. Lee Long \& R.G. Coles. 1995. Flood and cyclone related loss, and partial recovery of more than $1000 \mathrm{~km}^{2}$ of seagrass in Hervey Bay, Queensland, Australia. Aquat. Bot. 52: 3-17.

Ridler, M.S., R.C. Dent \& D.A. Arrington. 2006. Effects of Two Hurricanes on Syringodium filiforme, Manatee Grass, Within the Loxahatchee River Estuary, Southeast Florida. Estuaries and Coasts 29: 1019-1025.

Roger, C.S., G. Garrison, T. Grober, Z.M. Hillis \& M.A. Franke. 1994. Manual para el Monitoreo de Arrecifes de Coral en el Caribe y el Atlántico occidental: St. John, VI: National Park Service, Virgin Islands National Park.

Short, F.T. \& S. Wyllie-Echeverria. 1996. Natural and human induced disturbance of seagrasses. Environ. Conserv. 23: 17-27.

Steward, J.S., R.W. Virnstein, M.A. Lasi, L.J. Morris, J.D. Miller, L.M. Hall \& W.A. Tweedale. 2006. The Impacts of the 2004 Hurricanes on Hydrology, Water Quality, and Seagrass in the Central Indian River Lagoon, Florida. Estuaries and Coasts 29: 954-965.

Van Tussenbroek, B.I., M.G. Barba Santos, J.K. Van Dijk, M.S.N. Sanabria-Alcaraz \& M.L. Tellez-Calderon. 2008. Selective elimination of rooted plants from a tropical seagrass bed in a back-reef lagoon: a hypothesis tested by Hurricane Wilma (2005). West Palm Beach (Florida). J. Coastal Res. 24: 278-281.

Williams, S.L. 1990. Experimental studies of Caribbean seagrass bed development. Ecol. Monogr. 60: 449-469. 